\title{
Review
}

Molecular

Neuropsychiatry
Mol Neuropsychiatry 2016;2:37-45

DOI: $10.1159 / 000444755$
Received: October 7, 2015

Accepted: February 16, 2016

Published online: April 13, 2016

\section{A Review of Genome-Wide Association Studies of Stimulant and Opioid Use Disorders}

\author{
Kevin P. Jensen \\ Department of Psychiatry, Yale University School of Medicine, New Haven, Conn., and VA Connecticut Healthcare \\ System, West Haven, Conn., USA
}

\section{Key Words}

Opioid $\cdot$ Cocaine $\cdot$ Stimulant $\cdot$ Addiction $\cdot$ Genome-wide association studies

\begin{abstract}
Substance use disorders (SUD) are a major contributor to disability and disease burden worldwide. Risk for developing SUDs is influenced by variation in the genome. Identifying the genetic variants that influence SUD risk may help us to understand the biological mechanisms for the disorders and improve treatments. Genome-wide association studies (GWAS) have been successful in identifying many regions of the genome associated with common human disorders. Here, findings from recent GWAS of SUDs that involve illicit substances will be reviewed. Several GWAS have been reported, including studies on opioid and stimulant use disorder (cocaine and methamphetamine). Several of these GWAS report associations that are biologically interesting and statistically robust. Replication of the associations in independent samples and functional studies to understand the basis for the statistical associations will be important next steps.
\end{abstract}

(c) 2016 S. Karger AG, Basel

\section{KARGER}

E-Mail karger@karger.com

www.karger.com/mnp

\section{Introduction}

Illicit drug use can negatively affect an individual's health and well-being in profound ways [1]. The negative outcomes affect not only the individual, but also family, friends, associates and society. Illicit drug use is common. In a study of 10,904 randomly selected students attending college in the US in 2001, 12\% reported lifetime nonmedical use of prescription opioids, $7 \%$ reported use in the past year and 3\% reported use in the past month [2]. In a separate US sample, the lifetime prevalence of cocaine use was estimated to be as high as $16.2 \%$ [3]. This review will focus on the molecular genetics of substance use disorders (SUD) with a particular emphasis on opioids and stimulants (cocaine and methamphetamine). These are among the most commonly abused illicit drugs in the US (excluding cannabis, which has legal status at the state, but not federal, level in many parts of the US) [4]. The negative impact of their misuse is a major health concern and the focus of intense research.

One potential outcome of substance use is the development of an SUD. The clinical symptoms of an SUD include impairment or distress marked by the development of tolerance or withdrawal; substance use despite serious physical or psychological health problems; using more than intended, persistent unsuccessful efforts to reduce 
substance use; excessive time spent obtaining, using or recovering from substances and a pattern of use that negatively affects one's normal social, recreational or occupational activities [5].

Like many psychiatric disorders, SUDs have moderate heritability. The proportion of risk that can be attributed to heritable factors has been estimated with twin studies by comparing the rate of disorder concordance for dizygotic twins with that for monozygotic twins. A higher rate of concordance among monozygotic twins relative to dizygotic twins is evidence for genetic risk. Cocaine use disorder (CUD) risk for females is estimated to have heritability of 0.65 , while for males the estimated heritability is 0.79 [6]. Opioid use disorder (OUD) is also moderately heritable $[7,8]$. Although these genetic studies indicate that individual differences in genes have an important role in the development of SUDs, such differences are not deterministic; an individual's environment is a major factor. But identifying the genes that affect risk and characterizing their function is important because it could yield critical insight into the molecular basis for these disorders and potentially better ways to prevent or treat them.

Contemporary genome-wide association approaches have been successful in identifying many regions of the genome associated with human traits and disease, including psychiatric disorders. The success of these studies, with regard to reproducibility and biological insights gained, stands in stark contrast to prior candidate genedriven approaches. Among these genetic success stories are studies of bipolar disorder [9] and schizophrenia [10, $11]$, as well as several genome-wide association studies (GWAS) of legal drug use (alcohol, cigarette smoking and caffeine consumption) [12-15]. Combined, these studies have implicated a spectrum of genetic variation with effects on risk that range from barely detectable to moderately large. The genetic variation implicated in risk includes common single nucleotide polymorphisms (SNPs), rare variants and structural variants [e.g. copy number variants $(\mathrm{CNVs})]$. Genetic studies are only the initial step in the process to understanding the biological basis for these complex disorders. Rigorous replication of the genetic associations is necessary, and functional studies are required to understand the basis for the statistical associations.

GWAS for SUDs of legal drugs have yielded some results that are statistically robust and biologically interesting. For example, functional variants in an alcohol metabolism gene, alcohol dehydrogenase $1 \mathrm{~B}(A D H 1 B)$, are associated with alcohol use disorder (AUD) in several global populations based on genetic association studies that include GWAS [13, 16-18]. Individuals with a high activity form of this enzyme develop an aversive flushing reaction when they consume alcohol; these individuals are at low risk for developing AUD [19]. Thus, ethanol metabolism differences are a biologically plausible mechanism for AUD risk that is strongly supported by genetic association studies. Genome-wide approaches have little bias, and this creates the possibility of discovery of variants, genes and pathways with unanticipated biological involvement. For example, several studies, including large GWAS and GWAS meta-analyses of tobacco use disorder, have implicated in the regulation of smoking intensity (cigarettes smoked per day) a nonsynonymous SNP, rs16969968 (G>A; amino acid $398 \mathrm{D}>\mathrm{N})$, in the a5 nicotinic acetylcholine receptor gene (CHRNA5) [14, 15, $20-22]$. Subsequent in vitro and in vivo studies of $\alpha 5$ receptor function have characterized an intriguing mechanism that involves a previously unanticipated molecular pathway, namely a 5 signaling in the medial habenula and the regulation of aversion to nicotine [23-27]. In a sample of European American (EA) and African-American (AA) smokers, the CHRNA5 heavy smoking risk allele was associated with lower subjective ratings of aversive effects in response to intravenous infusions of nicotine [28]. These studies suggest a mechanism that might explain the association of CHRNA5 rs16969968 with smoking intensity. Discoveries that lead to unanticipated risk genes or pathways linked to new risk genes, provide biological insights that are especially important because they may accelerate the development of novel treatments anchored upon previously unsuspected mechanisms, which will yield great benefits to human health.

This article will review recent human molecular genetic studies of SUDs with an emphasis on opioid and stimulants, which are among the most commonly abused illicit drugs in the US. The review will focus on studies that employed a genome-wide approach given that genome-wide approaches can often lead to important biological insights into risk mechanisms that are unbiased by prior knowledge. The current findings will be discussed, as well as potential future directions.

\section{Literature Search Methods}

A literature search was performed in PubMed to identify abstracts of human studies with the terms 'genomewide' and 'association' in combination with 'cocaine', 'opioid', 'stimulant', 'illicit drugs', 'methamphetamine' or 'amphetamine'. Of the 56 studies identified by this search,
38

Mol Neuropsychiatry 2016;2:37-45 DOI: $10.1159 / 000444755$
Jensen 
Table 1. Genome-wide significant $\left(\mathrm{p}<5 \times 10^{-8}\right)$ findings from genome-wide association studies of opioid, stimulant and co-occurring substance use disorders

\begin{tabular}{|c|c|c|c|c|c|}
\hline First author, year [Ref.] & SUD phenotype & Method & Top variant & Nearest gene & Population \\
\hline \multicolumn{6}{|l|}{ Opioids } \\
\hline Gelernter, 2014 [29] & OUD (symptom count) & GWAS & rs62103177 & KCNG2 & $\mathrm{AA}$ \\
\hline $\mathrm{Li}, 2015[35]$ & OUD (case-control) & CNV-GWAS & $18 \mathrm{q} 12.3$ & LOC647946, KC6, PIK3C3 & $\mathrm{AA}, \mathrm{EA}$ \\
\hline \multicolumn{6}{|l|}{ Stimulants } \\
\hline \multicolumn{6}{|l|}{ Multiple } \\
\hline McGue, 2014 [61] & Illicit drug use & GWAS & rs1868152 & CRYGS & EA \\
\hline Johnson, 2015 [60] & Any SUD & GWAS & rs9829896 & $K A T 2 B$ & $\mathrm{AA}$ \\
\hline \multirow[t]{2}{*}{ Wetherill, 2015 [59] } & SUD (case-control) & GWAS & rs2952621 & LOC151121 & EA \\
\hline & SUD (symptom count) & GWAS & rs2567261 & ARHGAP28 & EA \\
\hline
\end{tabular}

$\mathrm{AU}=$ Australian

included in this review were 12 that used a genome-wide strategy and investigated opioid or stimulant use disorders or an SUD that included opioids or stimulants (cooccurring SUDs). Studies that investigated the response to opioids or stimulants in a clinical or laboratory setting were included if they also investigated effects on SUD risk in secondary analyses. Table 1 summarizes the results for studies that reported genome-wide significant $(\mathrm{p}<5 \times$ $10^{-8}$ ) SNP associations.

\section{GWAS of Opioid Sensitivity and OUD}

Several research groups have used genome-wide approaches to investigate the genetics of OUD. Gelernter et al. [29] conducted a GWAS of OUD risk involving three phases that cumulatively included $>12,000$ subjects. Most subjects were recruited from several eastern US recruitment sites, and additional subjects were accessed via publicly availably (dbGAP application) data from the Study of Addiction: Genetics and Environment (SAGE) [3033]. The total sample included 6,877 EA and 5,432 AA subjects. A standard OD diagnosis case-control design was employed, as well as a noteworthy symptom countbased analytic approach. This symptom count-based approach used as independent variables the DSM-IV symptom count for OUD, as well as the DSM-IV symptom count for three additional SUDs (cocaine, alcohol and nicotine), along with standard covariates; the minor allele dosage was the dependent variable. The symptom count trait design had several advantages, e.g. by using more of

Genome-Wide Association Studies of Stimulant and Opioid Use Disorders the available phenotypic data the statistical power was increased relative to the binary case-control design. Also, by including in the analysis model information on three additional SUDs (cocaine, alcohol and nicotine), comorbid substance use was controlled for, which favored the identification of genetic associations that might be specific to opioids rather than cocaine, alcohol and nicotine, or combinations of all. The most compelling results for this analysis derived from the analysis of OUD symptom count in the AA population. These results included several SNPs that mapped to KCNG2, which encodes a potassium voltage-gated ion channel. The top KCNG2 SNP, rs62103177, was genome-wide significant in the AA population after the results from all three AA analysis phases were combined by meta-analysis $\left(\mathrm{p}=3.6 \times 10^{-10}\right)$. Many of the top AA association signals, including rs62103177, were monomorphic in the EA samples, suggesting that these association findings could be population specific. In an extension of the GWAS phase of the study, Gelernter et al. [29] conducted a pathway analysis using the metaanalyzed results from each population. The most interesting results occurred within the AA population, where there was evidence for enrichment for calcium signaling [false discovery rate $(F D R)=0.15$ ] and long-term potentiation $(F D R=0.17)$ pathways. Another notable topranked pathway was for glucocorticoid receptor (GR) signaling $(\mathrm{FDR}=0.18)$.

Nelson et al. [34] reported a GWAS that compared heroin-dependent daily users $(\mathrm{n}=1167)$ with heroin-exposed subjects that had never progressed to daily heroin use $(n=161)$. The strongest association signals were SNPs 
that mapped to the cornichon family AMPA receptor auxiliary protein 3 gene (CNIH3), a glutamate receptorassociated regulatory protein encoded on chromosome 1. The associated SNPs were highly correlated and conditional analyses indicated that the association was likely driven by a single SNP at this locus. Several follow-up studies were initiated to support and extend these initial findings; foremost was a replication study in two independent samples of opioid-dependent case and control subjects. The first sample included subjects from the Gelernter et al. [29] genetic study of opioid dependence and the second sample included subjects from the SAGE [33]. After a meta-analysis of the three studies, the best statistical support for association was for $\operatorname{rs} 10799590$ ( $p=4.30$ $\left.\times 10^{-9}\right)$, with the effect for $r 10799590$ being consistent between the discovery sample $\left(\mathrm{p}=1.51 \times 10^{-6} ; \mathrm{OR}=0.55\right.$, $95 \%$ CI $0.43-0.70)$, the Gelernter study sample ( $\mathrm{p}=5.76$ $\left.\times 10^{-2} ; \mathrm{OR}=0.78,95 \% \mathrm{CI} 0.60-1.01\right)$ and the SAGE sample $\left(\mathrm{p}=6.73 \times 10^{-4} ; \mathrm{OR}=0.62,95 \%\right.$ CI $\left.0.47-0.82\right)$. Given that there were no CNIH3 exonic SNPs identified that were in high linkage disequilibrium with the identified OUD-associated SNPs, the potential effects of the SNPs on gene expression regulatory elements were explored. Interestingly, the best statistically supported SNP, rs10799590, mapped to a region of CNIH3 that was marked by an open chromatin state and enriched with interactions with modified histones. The epigenetic features associated with rs 10799590 were specific to DNA isolated from human fetal brain tissue, as they were not observed in several tissues that were of non-nervous system origin. To extend the findings further, Nelson et al. [34] investigated in an independent sample of EA subjects $(\mathrm{n}=312)$ the association of rs10799590 with amygdala habituation, an intermediate phenotype associated with psychopathology. The allele associated with low risk for OUD was associated with greater amygdala habituation in response to threat-related facial expressions. The final experiment investigated the association of haplotypes among 23 different inbred strains of mice with symptoms of physical dependence on morphine. CNIH3 haplotypes, as well as haplotypes for several glutamate-related signaling genes, were associated with naloxone-induced morphine withdrawal behavior. The study by Nelson et al. [34] presents compelling evidence for involvement of CNIH3 in the development of OUD.

Li et al. [35] took an alternative approach relative to the aforementioned studies that focused on SNP associations. Rather than focusing on SNPs, Li et al. [35] used genome-wide SNP data from genotyping microarrays to identify CNVs and investigated their association with
OUD. CNVs or other types of structural variation in the genome might explain some of the heritability for complex trait risk [36]. The study by Li et al. [35] included 5,152 subjects with genome-wide SNP array data (this sample overlaps partly with the sample used in the opioid study by Gelernter et al. [29]). To identify CNVs, Li et al. [35] used the raw intensity of GWAS array probes and the union of two CNV calling strategies, PennCNV [37] and QuantiSNP [38], and a third calling program, GNOSIS [39], was used to confirm CNVs identified by the first two programs. This approach ensured that there was a low false-positive discovery rate in the pool of CNVs that were used for association testing. Li et al. [35] first observed that in EA and AA samples, OUD cases had fewer CNVs than the controls. In a genome-wide case versus control association analysis of common CNVs (frequency $>1 \%$ ), the most robust statistical support was for an intergenic region on $18 \mathrm{q} 12.3$. There are several noncoding RNA transcripts adjacent to this CNV region (LOC647946, KC6). The nearest protein-coding gene is phosphatidylinositol 3-kinase catalytic subunit type 3 (PIK3C3), which is also known as VPS34. PIK3C3 encodes a broadly expressed regulator of intracellular trafficking and synaptic transmission that has an essential role in maintaining neuronal integrity [40]. CNVs at the $18 \mathrm{q} 12.3$ region (not necessarily the same CNVs as in the study by Li's group) have also been implicated in schizophrenia risk [41], and SNPs near the promoter region of PIK3C3 were found to be associated with bipolar disorder and schizophrenia [42]. Several additional regions of interest were identified in an analysis that focused on rare CNVs. Some of the CNVs of interest could potentially affect protein-coding genes that were previously reported to be associated with SUD, such as MAP3K4 with nicotine dependence [43], although as noted by Li et al. [35], most CNVs mapped to regions not previously implicated in OUD or SUD risk, and their functions are unknown. This important line of research requires more attention.

A study by Nishizawa et al. [44] focused on human opioid sensitivity using a multistage GWAS that started with a sample of 353 healthy Japanese subjects who had undergone elective cosmetic orthognathic surgery. The outcome they initially studied was patient-controlled use of the synthetic opioid fentanyl during the first $24 \mathrm{~h}$ after surgery. The initial sample was divided into subgroups with 118,117 and 118 subjects, respectively. SNPs with an association at $\mathrm{p}<0.05$ in the first sample were selected for association testing in the second sample, and SNPs with an association $p<0.05$ in the second sample were selected for association testing in the final sample. The top SNP
Jensen 
based on a meta-analysis of the results from the first, second and final analyses was rs2952768, which is located upstream from a protein-lysine methyltransferase gene METTL21A (also known as FAM119A) and the CAMPresponsive element-binding protein 1 gene (CREB1) on chromosome 2 . The rs2952768 minor allele (C) was associated with greater fentanyl use $\left(\mathrm{p}=8.044 \times 10^{-7}\right)$. In a second sample that consisted of 112 subjects who had undergone major abdominal surgery, rs2952768 was tested for association with postoperative analgesic use, although in this sample the analgesic was not necessarily fentanyl. Consistent with the initial analyses, $\mathrm{rs} 2952768^{*} \mathrm{C}$ homozygotes reported greater use of postoperative analgesics.

There were three interesting extensions of this initial finding that provided convergent support for a potential effect of rs2952768 on risk for OUD and other SUDs. Based on the hypothesis that decreased opioid sensitivity would be associated with lower drug dependence risk, rs2952768 was tested for association with several drug dependence traits. The low opioid sensitivity-associated allele (rs2952768*C) was associated with lower risks for (a) polydrug abuse in a methamphetamine dependence/ psychosis patient sample, (b) drug use in an alcohol-dependent sample and (c) drug and alcohol dependence in an eating disorder patient sample. In a separate sample of 500 healthy subjects, rs2952768 was tested for association with seven temperament dimensions assessed by the temperament and character inventory. Among the seven dimensions tested, the best statistically supported effect was observed for the 'reward dependence' dimension. The C-allele was associated with lower reward dependence scores, suggesting a mechanism for the previously observed associations with opioid sensitivity. A gene expression study was also conducted to test for rs 2952768 functional effects. In the gene expression study, rs2952768 was tested for association with mRNA expression of the two nearest genes, METTL21A (FAM119A) and CREB1, in postmortem brain tissue from 100 EA donors. In this analysis, rs2952768 was associated with CREB1 mRNA expression, but not METTL21A (FAM119A) mRNA expression. These findings are interesting in light of basic research linking CREB1 function to reward processes and the development of addiction [45-47]. This interesting study, based initially on analgesic opioid requirement in healthy subjects, yielded results that were generalizable to several related clinical and nonclinical SUD phenotypes.

An earlier GWAS conducted by Nielsen et al. [48] included 325 methadone-stabilized heroin-dependent subjects and 250 control subjects that were analyzed with the

Genome-Wide Association Studies of Stimulant and Opioid Use Disorders
Affymetrix GeneChip Mapping 100K Set. An SNP in an intergenic region on chromosome 1, rs10494334, was associated with heroin dependence in the EA subset after an experiment-wise significance test correction $(p=0.035)$.

\section{GWAS of Stimulant Use Disorders}

Gelernter et al. [29,49] reported a GWAS of CUD in a sample that overlapped with the previously described OUD GWAS. A similar symptom count-based analytic approach was used. The top statistically supported association was for CUD symptom count and rs2629540 (p = $4.28 \times 10^{-8}$ ). This SNP maps to an intron of the FAM53B ('family with sequence similarity 53 , member B') gene on chromosome 10. Support for this association came from EA and AA populations, and interestingly, the peak association signal mapped to within the 1-lod support interval of a previously identified CUD linkage peak [50]. In the linkage study, as in the GWAS, both EAs and AAs contributed to the finding [50]. These studies provide strong convergent support for a CUD risk allele at this locus. Although the function of FAM53B is not well understood in humans, a medaka (Oryzias latipes) gene homolog, simplet (smp), regulates cell proliferation [51], and in zebrafish (Danio rerio) this gene regulates axonal extension of a specific type of neurons in the embryo [52]. A study by Dickson et al. [53] identified quantitative trait loci (QTL) on chromosome 7 and 11 for a shift in the IV cocaine self-administration dose-response curve among several mouse strains that had acquired cocaine IV selfadministration. The chromosome 7 QTL was a genomewide significant trans-QTL for Fam53b gene mRNA expression in the midbrain of drug-naive mice. Although these functions are interesting, further work is required, including replication of the genetic associations, to understand FAM $53 B$ function in the mammalian nervous system and its relevance to CUD risk.

Uhl et al. [54] conducted a genetic association study of methamphetamine use disorder (MUD) in a sample of Japanese and a sample of Taiwanese case and matched control subjects. A pooled DNA genotyping strategy was used to compare genotype frequencies among MUD subjects with the control group. Two genes were identified, CDH13 and CSMD1, that had clusters of nominally associated SNPs that were associated with MUD, but no genome-wide significant single SNP association was reported. $\mathrm{CDH} 13$ has been implicated in the subjective response to stimulants ( $d$-amphetamine) in healthy volunteers [55]. The CUD GWAS by Gelernter et al. [49] 
reported a nominal association for a $C D H 13$ SNP, rs4782559, with cocaine-induced paranoia, a transient psychosis marked by episodes of paranoid delusions and hallucinations experienced by $>50 \%$ of chronic cocaine users [56].

Ikeda et al. [57] conducted a GWAS of methamphetamine dependence that included methamphetamine-dependent subjects $(\mathrm{n}=236)$ and healthy control subjects $(n=864)$ recruited from a geographically isolated area of Japan. Most of the methamphetamine-dependent subjects in this sample were included in the previous pooledsample GWAS by Uhl et al. [54]. Although no SNP surpassed the threshold for genome-wide significance, Ikeda et al. [57] found evidence for a genetic relationship between methamphetamine-induced psychosis and schizophrenia. There was an enrichment of methamphetamineinduced psychosis risk alleles in ethnically matched subjects with schizophrenia $[57,58]$. These findings are consistent with a growing body of evidence that indicates SUD traits are highly polygenic and risk variants have pleiotropic effects on psychiatric disorder risk.

\section{GWAS of Co-Occurring SUDs}

A large fraction of the genetic risk for SUDs is not specific to one particular substance; rather, the genetic risk is general to different types of substances with specificity being conferred by the environment [7]. To identify genes that might confer risk for any SUD, Wetherill et al. [59] conducted a GWAS of a binary and a quantitative measure of general substance dependence liability for alcohol, cannabis, cocaine or opioids in a sample of 2,322 EA subjects. The quantitative measure of general substance dependence liability was based on a factor analysis of DSMIV substance dependence symptoms. Two genomewide significant associations were observed. One SNP, rs2952621, near the end of the LOC151121 gene on chromosome 2 was associated with any substance dependence in a discovery sample $\left(\mathrm{p}=1.8 \times 10^{-8}\right)$ and in an independent replication sample $(\mathrm{p}=0.02)$. An SNP in ARHGAP28, which encodes Rho GTPase-activating protein 28 , was associated with the quantitative measure of substance dependence liability $\left(\mathrm{p}=3.8 \times 10^{-8}\right)$ in the discovery sample; however, this association was not supported in the replication sample $(\mathrm{p}=0.29)$.

Johnson et al. [60] conducted a GWAS of drug abuse in a sample that included 3,742 AA and 6,845 EA subjects. The majority of cases were recent opioid $(>60 \%)$ and cocaine $(>25 \%)$ users, while the control subjects included unassessed population controls and subjects that were negative for SUDs based on an assessment that used DSM-IV criteria. The case subjects were genotyped with the Omni1-Quad BeadChip (Illumina Inc., San Diego, Calif., USA), and control subjects were genotyped with the Omni1-Quad, 1 M-Duo or Omni2 BeadChip (Illumina). After quality control procedures, there were 2,017 AA cases and 1,725 AA controls and 1,142 EA cases and 5,703 EA controls. A replication sample that included 259 AA cases and 496 AA controls and 273 EA cases and 858 EA controls was assembled from a dataset available from dbGAP [Genetic Association Information Network (GAIN); accession number phs000021.v3.p2]. After imputations, $>6$ million markers were tested for association. In the discovery phase, one SNP, rs9829896, in KAT2B, was associated with drug abuse in the AA sample after genome-wide correction $\left(\mathrm{p}=4.63 \times 10^{-8}\right)$. The effect was also significant in the AA replication sample $(p=0.0019)$, and the strength of the association improved after a metaanalysis that combined the discovery and replication study results $\left(\mathrm{p}=3.93 \times 10^{-10}\right)$. There was no compelling evidence for a substance-specific effect of rs9829896, as the association signals were evident, albeit less robust, when opioid, cocaine, marijuana or amphetamine use disorders were analyzed separately. No significant effects were detected in the EA sample. In interesting follow-up studies, Johnson et al. [60] showed that rs9829896 was associated with $K A T 2 B$ mRNA expression in postmortem brains from AA subjects, but not EA subjects. A KAT2Bcentric network of 20 proteins that share pathways, physical interactions and protein domains was predicted. Messenger RNA expression for two proteins in the 20-protein network was associated with rs9829896 (MAML1, $\mathrm{p}=0.043 ;$ CREBBP, $\mathrm{p}=0.011)$ in AAs but not EAs.

GWAS of illicit drug use/misuse have also been reported in a sample of $\sim 7,100$ EA subjects that were participants in a longitudinal research project $[61,62]$. For each study, the illicit drug use phenotype was defined as a factor that was derived from the frequency of use of 11 different drugs (marijuana, amphetamines, barbiturates, tranquilizers, cocaine, heroin, opiates, PCP, psychedelics, inhalants and gas) and the symptoms of dependence [63]. The first study by McGue et al. [61] reported an association of rs1868152 near the CRYGS gene with illicit drug use $\left(\mathrm{p}=4.9 \times 10^{-8}\right)$. The second study by Vrieze et al. [62] was an exome-wide association study of a protein-coding rare variation genotyped by the Illumina HumanExome BeadChip. After quality control procedures, 111,592 nonsynonymous exonic variants were tested for associa-
Jensen 
tion with behavioral disinhibition and the use/misuse of nicotine, alcohol and illicit drugs. Although no single variant or gene-based associations were identified, heritability estimates based on the SNP data indicated that $35 \%$ of the heritability in the illicit drug use phenotype was explained by the rare nonsynonymous exonic variants. The percent of heritability explained by common and rare variants tagged by the arrayed SNPs was estimated to be $84 \%$ [62].

\section{Conclusions}

Recent GWAS of opioid, stimulant and co-occurring SUDs have identified several promising genes and pathways to guide future investigations on the biological mechanisms for SUDs. It is likely that more genes will emerge as sample sizes increase through the formation of large consortia, and the power to detect effects improves. In future studies, it will be important to include subjects that have had sufficient exposure to a substance (or substance class) for the potential development of an SUD. Establishing consensus exposure thresholds for the potential development of SUDs and gathering comprehensive substance use history will be important, although it may require a careful and thorough phenotypic assessment of subjects. Detailed information on substance use history could help determine whether any observed effects are specific or nonspecific to a particular substance (or substance class).

Genetic analysis methods, such as DNA sequencing, that capture more variation at higher resolution will help to fine-map association signals and identify putative 'casual' variants. Additional approaches that capture the aggregate effect of the many risk alleles that do not necessarily surpass the genome-wide significance threshold might also be used to investigate the molecular and behavioral effect of SUD genetic risk, for example the genetic relationship between SUDs and other disorders or traits and the effect of SUD risk genes on the acute response to drugs or treatment response. This type of approach has been informative for other traits, such as schizophrenia [64, 65].

Ultimately, replication studies and more focused studies in humans and animal models to determine the molecular and behavioral mechanisms for the observed statistical associations will be important next steps.

\section{Acknowledgements}

This work was supported by a US Department of Veteran Affairs VISN1 Career Development Award. The views expressed here do not reflect those of the Department of Veteran Affairs. Dr. Jensen would like to thank Dr. Joel Gelernter for his helpful comments and suggestions.

\section{Statement of Ethics}

There are no ethical issues to disclose.

\section{Disclosure Statement}

The author discloses that he has no conflict of interest.

\section{References}

1 Degenhardt L, Whiteford HA, Ferrari AJ, Baxter AJ, Charlson FJ, Hall WD, Freedman G, Burstein R, Johns N, Engell RE, Flaxman A, Murray CJ, and Vos T: Global burden of disease attributable to illicit drug use and dependence: findings from the Global Burden of Disease Study 2010. Lancet 2013;382:15641574.

2 McCabe SE, Teter CJ, Boyd CJ, Knight JR, Wechsler H: Nonmedical use of prescription opioids among U.S. college students: prevalence and correlates from a national survey. Addict Behav 2005;30:789-805.

3 Degenhardt L, Chiu WT, Sampson N, Kessler RC, et al: Toward a global view of alcohol, tobacco, cannabis, and cocaine use: findings from the WHO World Mental Health Surveys. PLoS Med 2008;5:1053-1067.

4 Degenhardt L, Hall W: Extent of illicit drug use and dependence, and their contribution to the global burden of disease. Lancet 2012; 379:55-70.

5 Diagnostic and Statistical Manual of Mental Disorders, ed 5. Washington, American Psychiatric Association, 2013.

6 Kendler KS, Prescott CA: Cocaine use, abuse and dependence in a population-based sample of female twins. Br J Psychiatry 1998;173: 345-350.

7 Kendler KS, Jacobson KC, Prescott CA, Neale MC: Specificity of genetic and environmental risk factors for use and abuse/dependence of cannabis, cocaine, hallucinogens, sedatives, stimulants, and opiates in male twins. Am J Psychiatry 2003;160:687-695.

8 Tsuang MT, Lyons MJ, Eisen SA, Goldberg J, True W, Lin N, Meyer JM, Toomey R, Faraone SV, Eaves L: Genetic influences on DSMIII-R drug abuse and dependence: a study of 3,372 twin pairs. Am J Med Genet 1996;67: 473-477.

9 Sklar P: Large-scale genome-wide association analysis of bipolar disorder identifies a new susceptibility locus near ODZ4 (vol 43, pg 977, 2011). Nat Genet 2012;44:1072-1072.

10 Biological insights from 108 schizophreniaassociated genetic loci. Nature 2014;511:421427.
Genome-Wide Association Studies of Stimulant and Opioid Use Disorders
Mol Neuropsychiatry 2016;2:37-45 DOI: $10.1159 / 000444755$ 
11 Genome-wide association study identifies five new schizophrenia loci. Nat Genet 2011; 43:969-976.

12 Coffee, Caffeine Genetics Consortium, Cornelis MC, Byrne EM, Esko T, et al: Genomewide meta-analysis identifies six novel loci associated with habitual coffee consumption. Mol Psychiatry 2015;20:647-656.

13 Gelernter J, Kranzler HR, Sherva R, Almasy L, Koesterer R, Smith AH, Anton R, Preuss UW, Ridinger M, Rujescu D, Wodarz N, Zill P, Zhao H, Farrer LA: Genome-wide association study of alcohol dependence: significant findings in African- and European-Americans including novel risk loci. Mol Psychiatry 2014; 19:41-49.

14 Thorgeirsson TE, Gudbjartsson DF, Surakka I, Vink JM, et al: Sequence variants at CHRNB3-CHRNA6 and CYP2A6 affect smoking behavior. Nat Genet 2010;42:448453.

15 Saccone NL, Culverhouse RC, Schwantes-An $\mathrm{TH}$, Cannon DS, et al: Multiple independent loci at chromosome 15q25.1 affect smoking quantity: a meta-analysis and comparison with lung cancer and COPD. PLoS Genet 2010;6.

16 Bierut LJ, Goate AM, Breslau N, Johnson EO, et al: ADH1B is associated with alcohol dependence and alcohol consumption in populations of European and African ancestry. Mol Psychiatry 2012;17:445-450.

17 Frank J, Cichon S, Treutlein J, Ridinger M, et al: Genome-wide significant association between alcohol dependence and a variant in the ADH gene cluster. Addict Biol 2012;17:171180.

18 Park BL, Kim JW, Cheong HS, Kim LH, Lee BC, Seo CH, Kang TC, Nam YW, Kim GB, Shin HD, Choi IG: Extended genetic effects of $\mathrm{ADH}$ cluster genes on the risk of alcohol dependence: from GWAS to replication. Hum Genet 2013;132:657-668.

19 Edenberg HJ: The genetics of alcohol metabolism: role of alcohol dehydrogenase and aldehyde dehydrogenase variants. Alcohol Res Health 2007;30:5-13.

20 Liu JZ, Tozzi F, Waterworth DM, Pillai SG, et al: Meta-analysis and imputation refines the association of $15 \mathrm{q} 25$ with smoking quantity. Nat Genet 2010;42:436-440.

21 Tobacco and Genetics Consortium: Genomewide meta-analyses identify multiple loci associated with smoking behavior. Nat Genet 2010;42:441-447.

22 Saccone SF, Hinrichs AL, Saccone NL, Chase GA, et al: Cholinergic nicotinic receptor genes implicated in a nicotine dependence association study targeting 348 candidate genes with 3713 SNPs. Hum Mol Genet 2007;16: 36-49.

23 Fowler CD, Lu Q, Johnson PM, Marks MJ, Kenny PJ: Habenular alpha5 nicotinic receptor subunit signalling controls nicotine intake. Nature 2011;471:597-601.
24 Frahm S, Slimak MA, Ferrarese L, SantosTorres J, Antolin-Fontes B, Auer S, Filkin S, Pons S, Fontaine JF, Tsetlin V, Maskos U, Ibanez-Tallon I: Aversion to nicotine is regulated by the balanced activity of beta 4 and alpha5 nicotinic receptor subunits in the medial habenula. Neuron 2011;70:522-535.

25 Jackson KJ, Marks MJ, Vann RE, Chen X, Gamage TF, Warner JA, Damaj MI: Role of alpha5 nicotinic acetylcholine receptors in pharmacological and behavioral effects of nicotine in mice. J Pharmacol Exp Ther 2010; 334:137-146.

26 Bierut LJ, Stitzel JA, Wang JC, Hinrichs AL, et al: Variants in nicotinic receptors and risk for nicotine dependence. Am J Psychiatry 2008; 165:1163-1171.

27 Kuryatov A, Berrettini W, Lindstrom J: Acetylcholine receptor (AChR) alpha5 subunit variant associated with risk for nicotine dependence and lung cancer reduces (alpha4beta2)(2)alpha5 AChR function. Mol Pharmacol 2011;79:119-125.

28 Jensen KP, DeVito EE, Herman AI, Valentine GW, Gelernter J, Sofuoglu M: A CHRNA5 smoking risk variant decreases the aversive effects of nicotine in humans. Neuropsychopharmacology 2015;40:2813-2821.

29 Gelernter J, Kranzler HR, Sherva R, Koesterer R, Almasy L, Zhao H, Farrer LA: Genomewide association study of opioid dependence: multiple associations mapped to calcium and potassium pathways. Biol Psychiatry 2014;76: 66-74.

30 Edenberg HJ: The collaborative study on the genetics of alcoholism: an update. Alcohol Res Health 2002;26:214-218.

31 Bierut LJ, Strickland JR, Thompson JR, Afful SE, Cottler LB: Drug use and dependence in cocaine dependent subjects, communitybased individuals, and their siblings. Drug Alcohol Depend 2008;95:14-22.

32 Bierut LJ: Genetic variation that contributes to nicotine dependence. Pharmacogenomics 2007;8:881-883.

33 Bierut LJ, Agrawal A, Bucholz KK, Doheny $\mathrm{KF}$, et al: A genome-wide association study of alcohol dependence. Proc Natl Acad Sci USA 2010;107:5082-5087.

34 Nelson EC, Agrawal A, Heath AC, Bogdan R, et al: Evidence of $\mathrm{CNIH} 3$ involvement in opioid dependence. Mol Psychiatry DOI: 10.1038/mp.2015.102.

35 Li D, Zhao H, Kranzler HR, Li MD, Jensen KP, Zayats T, Farrer LA, Gelernter J: Genome-wide association study of copy number variations (CNVs) with opioid dependence. Neuropsychopharmacology 2015;40:10161026.

36 Manolio TA, Collins FS, Cox NJ, Goldstein DB, et al: Finding the missing heritability of complex diseases. Nature 2009;461:747-753.
37 Wang K, Li M, Hadley D, Liu R, Glessner J, Grant SF, Hakonarson H, Bucan M: Penn CNV: An integrated hidden Markov model designed for high-resolution copy number variation detection in whole-genome SNP genotyping data. Genome Res 2007;17:16651674.

38 Colella S, Yau C, Taylor JM, Mirza G, Butler $\mathrm{H}$, Clouston P, Bassett AS, Seller A, Holmes CC, Ragoussis J: QuantiSNP: an objective Bayes Hidden-Markov model to detect and accurately map copy number variation using SNP genotyping data. Nucleic Acids Res 2007; 35:2013-2025.

39 Sanders SJ, Ercan-Sencicek AG, Hus V, Luo $\mathrm{R}$, et al: Multiple recurrent de novo CNVs, including duplications of the 7q11.23 Williams syndrome region, are strongly associated with autism. Neuron 2011;70:863-885.

40 Zhou X, Wang L, Hasegawa H, Amin P, Han BX, Kaneko S, He Y, Wang F: Deletion of $\mathrm{PIK} 3 \mathrm{C} 3 / \mathrm{Vps} 34$ in sensory neurons causes rapid neurodegeneration by disrupting the endosomal but not the autophagic pathway. Proc Natl Acad Sci USA 2010;107:9424-9429.

41 Glessner JT, Reilly MP, Kim CE, Takahashi N, et al: Strong synaptic transmission impact by copy number variations in schizophrenia. Proc Natl Acad Sci USA 2010;107:1058410589.

42 Stopkova P, Saito T, Papolos DF, Vevera J, Paclt I, Zukov I, Bersson YB, Margolis BA, Strous RD, Lachman HM: Identification of PIK3C3 promoter variant associated with bipolar disorder and schizophrenia. Biol Psychiatry 2004;55:981-988.

43 Grucza RA, Johnson EO, Krueger RF, Breslau N, Saccone NL, Chen LS, Derringer J, Agrawal A, Lynskey M, Bierut LJ: Incorporating age at onset of smoking into genetic models for nicotine dependence: evidence for interaction with multiple genes. Addict Biol 2010;15: 346-357.

44 Nishizawa D, Fukuda K, Kasai S, Hasegawa J, et al: Genome-wide association study identifies a potent locus associated with human opioid sensitivity. Mol Psychiatry 2014;19:5562.

45 Yan Z, Feng J, Fienberg AA, Greengard P: D-2 dopamine receptors induce mitogen-activated protein kinase and cAMP response element-binding protein phosphorylation in neurons. Proc Natl Acad Sci USA 1999;96: 11607-11612.

46 McClung CA, Nestler EJ: Regulation of gene expression and cocaine reward by CREB and Delta FosB. Nat Neurosci 2003;6:1208-1215.

47 Carlezon WA, Thome J, Olson VG, LaneLadd SB, Brodkin ES, Hiroi N, Duman RS, Neve RL, Nestler EJ: Regulation of cocaine reward by CREB. Science 1998;282:2272-2275.

48 Nielsen DA, Ji F, Yuferov V, Ho A, He CS, Ott J, Kreek MJ: Genome-wide association study identifies genes that may contribute to risk for developing heroin addiction. Psychiatr Genet 2010;20:207-214. 
49 Gelernter J, Sherva R, Koesterer R, Almasy L, Zhao H, Kranzler HR, Farrer L: Genomewide association study of cocaine dependence and related traits: FAM53B identified as a risk gene. Mol Psychiatry 2014;19:717-723.

50 Gelernter J, Panhuysen C, Weiss R, Brady K, Hesselbrock V, Rounsaville B, Poling J, Wilcox M, Farrer L, Kranzler HR: Genomewide linkage scan for cocaine dependence and related traits: significant linkages for a cocainerelated trait and cocaine-induced paranoia. Am J Med Genet B Neuropsychiatr Genet 2005; 136B:45-52.

51 Thermes V, Candal E, Alunni A, Serin G, Bourrat F, Joly JS: Medaka simplet (FAM53B) belongs to a family of novel vertebrate genes controlling cell proliferation. Development 2006;133:1881-1890.

52 Aoki $M$, Segawa $H$, Naito M, Okamoto $H$ : Identification of possible downstream genes required for the extension of peripheral axons in primary sensory neurons. Biochem Biophys Res Commun 2014;445:357-362.

53 Dickson PE, Miller MM, Calton MA, Bubier JA, Cook MN, Goldowitz D, Chesler EJ, Mittleman G: Systems genetics of intravenous cocaine self-administration in the BXD recombinant inbred mouse panel. Psychopharmacology (Berl) 2016;233:701-714.

54 Uhl GR, Drgon T, Liu QR, Johnson C, Walther D, Komiyama T, Harano M, Sekine Y, Inada T, Ozaki N, Iyo M, Iwata N, Yamada M, Sora I,
Chen CK, Liu HC, Ujike H, Lin SK: Genomewide association for methamphetamine dependence: convergent results from 2 samples. Arch Gen Psychiatry 2008;65:345-355.

55 Hart AB, Engelhardt BE, Wardle MC, Sokoloff G, Stephens M, de Wit H, Palmer AA: Genome-wide association study of $\mathrm{d}$-amphetamine response in healthy volunteers identifies putative associations, including cadherin 13 (CDH13). PLoS One 2012;7:e42646.

56 Brady KT, Lydiard RB, Malcolm R, Ballenger JC: Cocaine-induced psychosis. J Clin Psychiatry 1991;52:509-512.

57 Ikeda M, Okahisa Y, Aleksic B, Won M, Kondo N, Naruse N, Aoyama-Uehara K, Sora I, Iyo M, Hashimoto R, Kawamura Y, Nishida N, Miyagawa T, Takeda M, Sasaki T, Tokunaga K, Ozaki N, Ujike H, Iwata N: Evidence for shared genetic risk between methamphetamine-induced psychosis and schizophrenia. Neuropsychopharmacology 2013;38:18641870.

58 Ikeda M, Aleksic B, Kinoshita Y, Okochi T, et al: Genome-wide association study of schizophrenia in a Japanese population. Biol Psychiatry 2011;69:472-478.

59 Wetherill L, Agrawal A, Kapoor M, Bertelsen $\mathrm{S}$, et al: Association of substance dependence phenotypes in the COGA sample. Addict Biol 2015;20:617-627.

60 Johnson EO, Hancock DB, Levy JL, Gaddis NC, Page GP, Glasheen C, Saccone NL, Bierut
LJ, Kral AH: KAT2B polymorphism identified for drug abuse in African Americans with regulatory links to drug abuse pathways in human prefrontal cortex. Addict Biol DOI: 10.1111/adb.12286.

61 McGue M, Zhang Y, Miller MB, Basu S, Vrieze S, Hicks B, Malone S, Oetting WS, Iacono WG: A genome-wide association study of behavioral disinhibition. Behav Genet 2013;43:363-373.

62 Vrieze SI, Feng S, Miller MB, Hicks BM, Pankratz N, Abecasis GR, Iacono WG, McGue M: Rare nonsynonymous exonic variants in addiction and behavioral disinhibition. Biol Psychiatry 2014;75:783-789.

63 Hicks BM, Schalet BD, Malone SM, Iacono WG, McGue M: Psychometric and genetic architecture of substance use disorder and behavioral disinhibition measures for gene association studies. Behav Genet 2011;41:459475.

64 Purcell SM, Wray NR, Stone JL, Visscher PM, et al: Common polygenic variation contributes to risk of schizophrenia and bipolar disorder. Nature 2009;460:748-752.

65 Frank J, Lang M, Witt SH, Strohmaier J, Rujescu D, Cichon S, Degenhardt F, Nothen MM, Collier DA, Ripke S, Naber D, Rietschel $\mathrm{M}$ : Identification of increased genetic risk scores for schizophrenia in treatment-resistant patients. Mol Psychiatry 2015;20:150151 